\title{
HYPERBOLIC HEMIVARIATIONAL INEQUALITIES WITH BOUNDARY SOURCE AND DAMPING TERMS
}

\author{
Jin Mun Jeong, Jong Yeoul Park, and Sun Hye Park
}

\begin{abstract}
In this paper we study the existence of global weak solutions for a hyperbolic hemivariational inequalities with boundary source and damping terms, and then investigate the asymptotic stability of the solutions by using Nakao Lemma [8].
\end{abstract}

\section{Introduction}

In this paper, we are concerned with the global existence and the asymptotic stability of weak solutions for a hyperbolic hemivariational inequality with nonlinear damping and source terms on the boundary:

$$
\left\{\begin{array}{l}
y_{t t}-\Delta y_{t}-\operatorname{div}\left(|\nabla y|^{p-2} \nabla y\right)=0 \text { in } \Omega \times(0, \infty), \\
y=0 \text { on } \Gamma_{1} \times(0, \infty), \\
\frac{\partial y_{t}}{\partial \nu}+\nu \cdot\left(|\nabla y|^{p-2} \nabla y\right)+\Xi=|y|^{m-2} y \text { on } \Gamma_{0} \times(0, \infty), \\
\Xi(x, t) \in \varphi\left(y_{t}(x, t)\right) \text { a.e. }(x, t) \in \Gamma_{0} \times(0, \infty), \\
y(x, 0)=y_{0}(x), y_{t}(x, 0)=y_{1}(x) \text { in } x \in \Omega
\end{array}\right.
$$

where $\Omega$ is a bounded domain in $\mathbb{R}^{N}$ with sufficiently smooth boundary $\Gamma:=\partial \Omega$ such that $\Gamma=\Gamma_{0} \cup \Gamma_{1}, \overline{\Gamma_{0}} \cap \overline{\Gamma_{1}}=\emptyset, \Gamma_{0}$ and $\Gamma_{1}$ have positive measures, $y_{t}=\frac{\partial y}{\partial t}$, $y_{t t}=\frac{\partial^{2} y}{\partial t^{2}}, \nu$ is the unit outward normal vector to $\Gamma$ and $\varphi$ is a discontinuous and nonlinear set valued mapping by filling in jumps of a locally bounded function $b$. The precise hypotheses on the above system will be given in the next section.

Recently, a class of hemivariational inequalities are studied by many authors $[2,6,7,11,14,15,16,19]$. Most of them considered the existence of weak solutions for differential inclusions of various forms. Miettinen and Panagiotopoulos $[6,7]$ proved the existence of weak solutions for some parabolic hemivariational inequalities. Park et al. [14] showed the existence of a global weak solution to the hyperbolic hemivariational inequality with Dirichlet boundary condition and without source term by making use of the Faedo-Galerkin approximation,

Received February 11, 2008.

2000 Mathematics Subject Classification. 35L85, 35Q72, 49 J53.

Key words and phrases. hemivariational inequality, existence of solution, asymptotic stability, source term.

This work was supported by the Korea Research Foundation Grant funded by the Korean Government (MOEHRD, Basic Research Promotion Fund) (KRF-2007-355-C00002). 
and then considered asymptotic stability of the solution by using Nakao Lemma [8] and Parks [15] investigated uniform decay rates of the solutions for a hyperbolic system with differential inclusion and memory source terms on the boundary by using the perturbed method.

The background of these variational problems are in physics, especially in solid mechanics, where nonconvex, nonmonotone and multi-valued constitutive laws lead to differential inclusions. We refer to $[11,12]$ to see the applications of differential inclusions. On the other hand, it is interesting to mention that the existence and nonexistence of global solutions for nonlinear wave equations with nonlinear damping and source terms in a bounded domain have been studied by many authors $[4,5,10,13,18]$ in the past twenty years. Thus, in this paper we shall deal with the existence and the asymptotic behavior of a global weak solution for the hyperbolic hemivariational inequality (1.1) involving $p$-Laplacian, a nonlinear, discontinuous and multi-valued damping and nonlinear source terms on the boundary. As far as we are concerned there is a little literature dealing with asymptotic behavior of solutions for hemivariational inequalities with boundary source terms as studied in this paper. The difficulties come from the interaction between the $p$-Laplacian and boundary source terms.

The plan of this paper is as follows. In Section 2, the main results besides notations and assumptions are stated. In Section 3, the existence of global weak solutions to the problem (1.1) is proved by using the potential well method and the Faedo-Galerkin method. In Section 4, the asymptotic stability of the solutions is investigated by using Nakao lemma.

\section{Statement of main results}

Throughout this paper we denote

$$
\begin{gathered}
V=\left\{y \in W^{1, p}(\Omega): y=0 \text { on } \Gamma_{1}\right\} \\
(y, z)=\int_{\Omega} y(x) z(x) d x, \quad(y, z)_{\Gamma_{0}}=\int_{\Gamma_{0}} y(x) z(x) d \Gamma .
\end{gathered}
$$

For every $q \in(1, \infty)$, we denote $\|\cdot\|_{q}=\|\cdot\|_{L^{q}(\Omega)},\|\cdot\|_{q, \Gamma_{0}}=\|\cdot\|_{L^{q}\left(\Gamma_{0}\right)}$. For simplicity, we denote $\|\cdot\|_{2},\|\cdot\|_{2, \Gamma_{0}}$ by $\|\cdot\|,\|\cdot\|_{\Gamma_{0}}$, respectively. For a Banach space $X$, we denote $\|\cdot\|_{X}$ the norm of $X$.

We assume that $p$ and $m$ are positive real numbers satisfying

$$
2 \leq p<m<\frac{(N-1) p}{2(N-p)}+1(2 \leq p<m<\infty \text { if } p=N) .
$$

Define the potential well

$$
\mathcal{W}=\left\{y \in V \mid I(y)=\|\nabla y\|_{p}^{p}-\|y\|_{m, \Gamma_{0}}^{m}>0\right\} \cup\{0\} .
$$

Then $\mathcal{W}$ is a neighborhood of 0 in $V$. Indeed, Sobolev imbedding (see [1])

$$
V \hookrightarrow L^{m}\left(\Gamma_{0}\right)
$$


and Poincare's inequality yield

$$
\|y\|_{m, \Gamma_{0}}^{m} \leq c_{*}^{m}\|\nabla y\|_{p}^{m} \leq c_{*}^{m}\|\nabla y\|_{p}^{m-p}\|\nabla y\|_{p}^{p}, \quad \forall y \in V,
$$

where $c_{*}$ is an imbedding constant from $V$ to $L^{m}\left(\Gamma_{0}\right)$. From this we deduce that $I(y)>0$ (i.e., $y \in \mathcal{W})$ if $\|\nabla y\|_{p}<\left(c_{*}^{-m}\right)^{1 /(m-p)}$.

For later purpose, we introduce the functional $J$ defined by

$$
J(y):=\frac{1}{p}\|\nabla y\|_{p}^{p}-\frac{1}{m}\|y\|_{m, \Gamma_{0}}^{m} .
$$

Obviously, we have

$$
J(y)=\frac{1}{m} I(y)+\frac{m-p}{m p}\|\nabla y\|_{p}^{p}
$$

Define $A: V \rightarrow V^{*}$ by

$$
\langle A y, z\rangle=\left(|\nabla y|^{p-2} \nabla y, \nabla z\right) \text { for all } z \in V,
$$

where $V^{*}$ denotes the dual space of $V$ and $\langle\cdot$,$\rangle the dual pairing between V$ and $V^{*}$.

Then the operator $A$ is bounded, monotone, hemicontinuous (see e.g. [3]) and

$$
\langle A y, y\rangle=\|\nabla y\|_{p}^{p},\left\langle A y, y_{t}\right\rangle=\frac{1}{p} \frac{d}{d t}\|\nabla y\|_{p}^{p} \text { for } y \in V .
$$

Now, we formulate the following assumptions:

$\left(H_{1}\right)$ Let $b: \mathbb{R} \rightarrow \mathbb{R}$ be a locally bounded function satisfying

$$
b(s) s \geq \mu_{1} s^{2} \text { and }|b(s)| \leq \mu_{2}|s| \text { for } s \in \mathbb{R},
$$

where $\mu_{1}$ and $\mu_{2}$ are some positive constants.

$\left(H_{2}\right) y_{0} \in \mathcal{W}, y_{1} \in L^{2}(\Omega)$ and

$$
\begin{aligned}
0 & <E(0)=\frac{1}{2}\left\|y_{1}\right\|^{2}+\frac{1}{p}\left\|\nabla y_{0}\right\|_{p}^{p}-\frac{1}{m}\left\|y_{0}\right\|_{m, \Gamma_{0}}^{m} \\
& <\frac{m-p}{2 m p}\left(\frac{m-p}{c_{*}^{m} 2(m-1) p}\right)^{p /(m-p)} .
\end{aligned}
$$

The multi-valued function $\varphi: \mathbb{R} \rightarrow 2^{\mathbb{R}}$ is obtained by filling in jumps of a function $b: \mathbb{R} \rightarrow \mathbb{R}$ by means of the functions $\underline{b}_{\epsilon}, \bar{b}_{\epsilon}, \underline{b}, \bar{b}: \mathbb{R} \rightarrow \mathbb{R}$ as follows

$$
\begin{aligned}
& \underline{b}_{\epsilon}(t)=\underset{|s-t| \leq \epsilon}{\operatorname{essinf}} b(s), \quad \bar{b}_{\epsilon}(t)=\underset{|s-t| \leq \epsilon}{\operatorname{ess} \sup } b(s) ; \\
& \underline{b}(t)=\lim _{\epsilon \rightarrow 0^{+}} b_{\epsilon}(t), \quad \bar{b}(t)=\lim _{\epsilon \rightarrow 0^{+}} \bar{b}_{\epsilon}(t) ; \\
& \varphi(t)=[\underline{b}(t), \bar{b}(t)] .
\end{aligned}
$$

We shall need a regularization of $b$ defined by

$$
b^{n}(t)=n \int_{-\infty}^{\infty} b(t-\tau) \rho(n \tau) d \tau
$$


where $\rho \in C_{0}^{\infty}((-1,1)), \rho \geq 0$ and $\int_{-1}^{1} \rho(\tau) d \tau=1$. It is easy to show that $b^{n}$ is continuous for all $n \in \mathbb{N}$ and $\underline{b}_{\epsilon}, \bar{b}_{\epsilon}, \underline{b}, \bar{b}, b^{n}$ satisfy the same condition $\left(H_{1}\right)$ with a possibly different constant if $b$ satisfies $\left(H_{1}\right)$. So, in the sequel, we denote the different constant by the same symbol as the original constants.

Definition. A function $y(x, t)$ is a weak solution to problem (1.1) if for every $T>0, y$ satisfies $y \in L^{\infty}(0, T ; V), y_{t} \in L^{2}\left(0, T ; W^{1,2}(\Omega)\right) \cap L^{\infty}\left(0, T ; L^{2}(\Omega)\right)$, $y_{t t} \in L^{2}\left(0, T ; V^{*}\right)$, there exists $\Xi \in L^{2}\left(0, T ; L^{2}\left(\Gamma_{0}\right)\right)$ and the following relations hold:

$$
\begin{aligned}
& \int_{0}^{T}\left\{\left\langle y_{t t}(t), z\right\rangle+\left(\nabla y_{t}(t), \nabla z\right)+\left(|\nabla y(t)|^{p-2} \nabla y(t), \nabla z\right)+(\Xi(t), z)_{\Gamma_{0}}\right\} d t \\
= & \int_{0}^{T}\left(|y(t)|^{m-2} y(t), z\right)_{\Gamma_{0}} d t, \forall z \in V, \\
& \Xi(x, t) \in \varphi\left(y_{t}(x, t)\right) \text { a.e. }(x, t) \in \Sigma_{0}:=\Gamma_{0} \times(0, T), \\
& y(0)=y_{0}, y_{t}(0)=y_{1} .
\end{aligned}
$$

Theorem 2.1. Under the assumptions $\left(H_{1}\right)$ and $\left(H_{2}\right)$ the problem $(1.1)$ has a weak solution.

Theorem 2.2. Under the same conditions of Theorem 2.1, the solutions of problem (1.1) satisfy the following decay rates:

If $p=2$, then there exist positive constants $C$ and $\gamma$ such that

$$
E(t) \leq C \exp (-\gamma t) \text { a.e. } t \geq 0,
$$

and if $p>2$, then there exists a constant $C>0$ such that

$$
E(t) \leq C(1+t)^{-p /(p-2)} \text { a.e. } t \geq 0,
$$

where $E(t)=\frac{1}{2}\left\|y_{t}(t)\right\|^{2}+\frac{1}{p}\|\nabla y(t)\|_{p}^{p}-\frac{1}{m}\|y(t)\|_{m, \Gamma_{0}}^{m}$.

In order to prove the decay rates of Theorem 2.2, we need the following lemma by Nakao (see $[8,9]$ for the proof) :

Lemma 2.1. Let $\phi: \mathbb{R}^{+} \rightarrow \mathbb{R}$ be a bounded nonincreasing and nonnegative function for which there exist constants $\alpha>0$ and $\beta \geq 0$ such that

$$
\sup _{t \leq s \leq t+1}(\phi(s))^{1+\beta} \leq \alpha(\phi(t)-\phi(t+1)), \forall t \geq 0 .
$$

Then

(1) If $\beta=0$, there exist positive constants $C$ and $\gamma$ such that

$$
\phi(t) \leq C \exp (-\gamma t), \quad \forall t \geq 0 .
$$

(2) If $\beta>0$, there exists a positive constant $C$ such that

$$
\phi(t) \leq C(1+t)^{-1 / \beta}, \forall t \geq 0 .
$$




\section{Proof of Theorem 2.1}

In this section we are going to show the existence of solutions to the problem (1.1) using the Faedo-Galerkin approximation and the potential method. To this end let $\left\{w_{j}\right\}_{j=1}^{\infty}$ be a basis in $V$ which are orthogonal in $L^{2}(\Omega)$. Let $V_{n}=$ $\operatorname{Span}\left\{w_{1}, w_{2}, \ldots, w_{n}\right\}$. We choose $y_{0}^{n}$ and $y_{1}^{n}$ in $V_{n}$ such that

$$
y_{0}^{n} \rightarrow y_{0} \text { in } V \text { and } y_{1}^{n} \rightarrow y_{1} \text { in } L^{2}(\Omega) \text {. }
$$

Let $y^{n}(t)=\sum_{j=1}^{n} g_{j n}(t) w_{j}$ be the solution to the approximate equation

$$
\left\{\begin{array}{c}
\left(y_{t t}^{n}(t), w_{j}\right)+\left(\nabla y_{t}^{n}(t), \nabla w_{j}\right)+\left\langle A y^{n}(t), w_{j}\right\rangle \\
+\left(b^{n}\left(y_{t}^{n}(t)\right), w_{j}\right) \Gamma_{0}=\left(\left|y^{n}(t)\right|^{m-2} y^{n}(t), w_{j}\right)_{\Gamma_{0}} \\
y^{n}(0)=y_{0}^{n}, y_{t}^{n}(0)=y_{1}^{n}
\end{array}\right.
$$

By standard methods of ordinary differential equations, we can prove the existence of a solution to $(3.2)$ on some interval $\left[0, t_{m}\right)$. Then this solution can be extended to the closed interval $[0, T]$ by using the a priori estimate below.

Step 1 : A priori estimate. Eq.(3.1) and the condition $y_{0} \in \mathcal{W}$ imply that

$$
I\left(y_{0}^{n}\right)=\left\|\nabla y_{0}^{n}\right\|_{p}^{p}-\left\|y_{0}^{n}\right\|_{m, \Gamma_{0}}^{m} \rightarrow I\left(y_{0}\right)>0
$$

Hence, without loss of generality, we assume that $I\left(y_{0}^{n}\right)>0$ (i.e., $y_{0}^{n} \in \mathcal{W}$ ) for all $n$. Substituting $w_{j}$ in $(3.2)$ by $y_{t}^{n}(t)$, we obtain

$$
\frac{d}{d t} E^{n}(t)+\left\|\nabla y_{t}^{n}(t)\right\|^{2}+\left(b^{n}\left(y_{t}^{n}(t)\right), y_{t}^{n}(t)\right)_{\Gamma_{0}}=0
$$

where

$$
\begin{aligned}
E^{n}(t) & =\frac{1}{2}\left\|y_{t}^{n}(t)\right\|^{2}+\frac{1}{p}\left\|\nabla y^{n}(t)\right\|_{p}^{p}-\frac{1}{m}\left\|y^{n}(t)\right\|_{m, \Gamma_{0}}^{m} \\
& =\frac{1}{2}\left\|y_{t}^{n}(t)\right\|^{2}+J\left(y^{n}(t)\right)
\end{aligned}
$$

Integrating (3.3) over $(0, \mathrm{t})$ and using the assumption $\left(H_{1}\right)$, we have

$$
\frac{1}{2}\left\|y_{t}^{n}(t)\right\|^{2}+J\left(y^{n}(t)\right)+\int_{0}^{t}\left\|\nabla y_{t}^{n}(\tau)\right\|^{2} d \tau \leq E^{n}(0)
$$

Since $E^{n}(0) \rightarrow E(0)$ and $E(0)>0$, without loss of generality, we assume that $E^{n}(0)<2 E(0)$ for all $n$. Now, we claim

$$
y^{n}(t) \in \mathcal{W}, t>0 .
$$

Assume that there exists a constant $T>0$ such that $y^{n}(t) \in \mathcal{W}$ for $t \in[0, T)$ and $y^{n}(T) \in \partial \mathcal{W}$, i.e., $I\left(y^{n}(T)\right)=0$. From (2.3), (3.3), and (3.4), we obtain

$$
J\left(y^{n}(T)\right)=\frac{m-p}{p m}\left\|\nabla y^{n}(T)\right\|_{p}^{p} \leq E^{n}(T) \leq E^{n}(0)<2 E(0)
$$

and therefore

$$
\left\|\nabla y^{n}(T)\right\|_{p}<\left(\frac{2 p m}{m-p} E(0)\right)^{1 / p}
$$


Combining this with (2.2) and using (2.4), we see that

$$
\begin{aligned}
\left\|y^{n}(T)\right\|_{m, \Gamma_{0}}^{m} & <c_{*}^{m}\left(\frac{2 p m}{m-p} E(0)\right)^{(m-p) / p}\left\|\nabla y^{n}(T)\right\|_{p}^{p} \\
& <\frac{m-p}{2(m-1) p}\left\|\nabla y^{n}(T)\right\|_{p}^{p}<\left\|\nabla y^{n}(T)\right\|_{p}^{p}
\end{aligned}
$$

where we used the fact that $(m-p) / 2(m-1) p<1$. This gives $I\left(y^{n}(T)\right)>0$, which is a contradiction. Therefore (3.6) is valid. From (2.3), (3.5), and (3.6),

$$
\frac{1}{2}\left\|y_{t}^{n}(t)\right\|^{2}+\frac{m-p}{p m}\left\|\nabla y^{n}(t)\right\|_{p}^{p}+\int_{0}^{t}\left\|\nabla y_{t}^{n}(s)\right\|^{2} d s<2 E(0) .
$$

By $\left(H_{1}\right)$, trace theorem and $(3.7)$, it follows that

$$
\int_{0}^{t}\left\|b^{n}\left(y_{t}^{n}(s)\right)\right\|_{\Gamma_{0}}^{2} d s \leq \mu_{2}^{2} \int_{0}^{t}\left\|y_{t}^{n}(s)\right\|_{\Gamma_{0}}^{2} d s \leq c \int_{0}^{t}\left\|\nabla y_{t}^{n}(s)\right\|^{2} d s \leq c E(0)
$$

here and in the sequel we denote by $c$ a generic positive constant independent of $n$ and $t$.

It follows from (3.7) and (3.8) that

$$
\left\{\begin{array}{l}
\left(y^{n}\right) \text { is bounded in } L^{\infty}(0, T ; V), \\
\left(y_{t}^{n}\right) \text { is bounded in } L^{\infty}\left(0, T ; L^{2}(\Omega)\right) \cap L^{2}\left(0, T ; W^{1,2}(\Omega)\right), \\
\left(b^{n}\left(y_{t}^{n}\right)\right) \text { is bounded in } L^{2}\left(0, T ; L^{2}\left(\Gamma_{0}\right)\right)
\end{array}\right.
$$

and since $A: V \rightarrow V^{*}$ is a bounded operator, it follows from (3.9) that

$$
\left(A y^{n}\right) \text { is bounded in } L^{\infty}\left(0, T ; V^{*}\right) \text {. }
$$

Finally we will obtain an estimate for $y_{t t}^{n}$. Since the imbedding $V \hookrightarrow L^{m}\left(\Gamma_{0}\right)$ is continuous, we have

$$
\left|\left(\left|y^{n}(t)\right|^{m-2} y^{n}(t), z\right)_{\Gamma_{0}}\right| \leq\left\|y^{n}(t)\right\|_{m, \Gamma_{0}}^{m-1}\|z\|_{m, \Gamma_{0}} \leq c\left\|y^{n}(t)\right\|_{1, p}^{m-1}\|z\|_{1, p}
$$

From (3.2), it follows that

$$
\begin{aligned}
\left|\int_{0}^{T}\left(y_{t t}^{n}(t), z\right) d t\right| \leq & \int_{0}^{T} \mid-\left\langle A y^{n}(t), z\right\rangle-\left(\nabla y_{t}^{n}(t), \nabla z\right) \\
& -\left(b^{n}\left(y_{t}^{n}(t)\right), z\right)_{\Gamma_{0}}+\left(\left|y^{n}(t)\right|^{m-2} y^{n}(t), z\right)_{\Gamma_{0}} \mid d t, \quad \forall z \in V_{m}
\end{aligned}
$$

and hence we obtain from (3.9)-(3.11) that

$$
\int_{0}^{T}\left\|y_{t t}^{n}(t)\right\|_{V^{*}}^{2} d t \leq c .
$$


Step 2: Passage to the limit. From (3.9), (3.10) and (3.12), we can extract a subsequence from $\left\{y^{n}\right\}$, still denoted by $\left\{y^{n}\right\}$, such that

$$
\left\{\begin{array}{l}
y^{n} \rightarrow y \text { weakly star in } L^{\infty}(0, T ; V) \\
y_{t}^{n} \rightarrow y_{t} \text { weakly in } L^{2}\left(0, T ; W^{1,2}(\Omega)\right), \\
y_{t}^{n} \rightarrow y_{t} \text { weakly star in } L^{\infty}\left(0, T ; L^{2}(\Omega)\right) \\
y_{t t}^{n} \rightarrow y_{t t} \text { weakly in } L^{2}\left(0, T ; V^{*}\right) \\
A y^{n} \rightarrow \zeta \text { weakly star in } L^{\infty}\left(0, T ; V^{*}\right), \\
b^{n}\left(y^{n}\right) \rightarrow \Xi \text { weakly star in } L^{2}\left(0, T ; L^{2}\left(\Gamma_{0}\right)\right) .
\end{array}\right.
$$

Considering that the imbeddings $V \hookrightarrow L^{2}(\Omega)$ and $W^{1,2}(\Omega) \hookrightarrow L^{2}\left(\Gamma_{0}\right)$ are compact and using the Aubin-Lions compactness lemma [3], it follows from (3.13) that

$$
\begin{aligned}
& y^{n} \rightarrow y \text { strongly in } L^{2}\left(0, T ; L^{2}(\Omega)\right) \cap L^{2}\left(0, T ; L^{2}\left(\Gamma_{0}\right)\right), \\
& y_{t}^{n} \rightarrow y_{t} \text { strongly in } L^{2}\left(0, T ; L^{2}\left(\Gamma_{0}\right)\right) .
\end{aligned}
$$

Using the first convergence result in (3.13) and the fact that the imbedding $V \hookrightarrow L^{2(m-1)}\left(\Gamma_{0}\right)\left(p<m<\frac{(N-1) p}{2(N-p)}+1\right.$ if $N>p$ and $p<m<\infty$ if $\left.p=N\right)$ is continuous, we obtain

$$
\left\|\left|y^{n}\right|^{m-2} y^{n}\right\|_{L^{2}\left(\Sigma_{0}\right)}^{2}=\int_{0}^{T} \int_{\Gamma_{0}}\left|y^{n}(x, t)\right|^{2(m-1)} d x d t \leq c .
$$

This implies that

$$
\left|y^{n}\right|^{m-2} y^{n} \rightarrow \xi \text { weakly in } L^{2}\left(\Sigma_{0}\right) .
$$

On the other hand, we have from (3.14) that $y^{n}(x, t) \rightarrow y(x, t)$ a.e. in $\Sigma_{0}$ and thus $\left|y^{n}(x, t)\right|^{m-2} y^{n}(x, t) \rightarrow|y(x, t)|^{m-2} y(x, t)$ a.e. in $\Sigma_{0}$. Therefore we conclude from (3.16) that $\xi(x, t)=|y(x, t)|^{m-2} y(x, t)$ a.e. in $\Sigma_{0}$.

Letting $n \rightarrow \infty$ in (3.2) and using the convergence results above, we have

$$
\begin{aligned}
& \int_{0}^{T}\left\{\left\langle y_{t t}(t), z\right\rangle+\left(\nabla y_{t}(t), \nabla z\right)+(\zeta(t), z)+(\Xi(t), z)_{\Gamma_{0}}\right\} d t \\
= & \int_{0}^{T}\left(|y(t)|^{m-2} y(t), z\right)_{\Gamma_{0}} d t, \quad \forall z \in V .
\end{aligned}
$$

Step 3: $(y, \Xi)$ is a solution of (1.1). Let $\phi \in C^{1}[0, T]$ with $\phi(T)=0$. By replacing $w_{j}$ by $\phi(t) w_{j}$ in (3.2) and integrating by parts the result over $(0, T)$, we have

$$
\begin{aligned}
& \left(y_{t}^{n}(0), \phi(0) w_{j}\right)+\int_{0}^{T}\left(y_{t}^{n}(t), \phi_{t}(t) w_{j}\right) d t \\
= & \int_{0}^{T}\left(\nabla y_{t}^{n}(t), \phi(t) \nabla w_{j}\right) d t+\int_{0}^{T}\left\langle A y^{n}(t), \phi(t) w_{j}\right\rangle d t \\
& +\int_{0}^{T}\left(b^{n}\left(y_{t}^{n}(t)\right), \phi(t) w_{j}\right)_{\Gamma_{0}} d t-\int_{0}^{T}\left(\left|y^{n}(t)\right|^{m-2} y^{n}(t), \phi(t) w_{j}\right)_{\Gamma_{0}}
\end{aligned}
$$


Similarly from (3.17) we get

$$
\begin{aligned}
& \left(y_{t}(0), \phi(0) w_{j}\right)+\int_{0}^{T}\left(y_{t}(t), \phi_{t}(t) w_{j}\right) d t \\
= & \int_{0}^{T}\left(\nabla y_{t}(t), \phi(t) \nabla w_{j}\right) d t+\int_{0}^{T}\left\langle\zeta(t), \phi(t) w_{j}\right\rangle d t \\
& +\int_{0}^{T}\left(\Xi(t), \phi(t) w_{j}\right)_{\Gamma_{0}} d t-\int_{0}^{T}\left(|y(t)|^{m-2} y(t), \phi(t) w_{j}\right)_{\Gamma_{0}} .
\end{aligned}
$$

Comparing between (3.18) and (3.19) we infer that

$$
\lim _{n \rightarrow \infty}\left(y_{t}^{n}(0)-y_{t}(0), w_{j}\right)=0, j=1,2, \ldots
$$

This implies that $y_{t}^{n}(0) \rightarrow y_{t}(0)$ weakly in $V^{*}$. By the uniqueness of limit, $y_{t}(0)=y_{1}$. Analogously, taking $\phi \in C^{2}[0, T]$ with $\phi(T)=\phi^{\prime}(T)=0$, we can obtain that $y(0)=y_{0}$.

Now, we show that $\Xi(x, t) \in \varphi\left(y_{t}(x, t)\right)$ a.e. in $\Sigma_{0}$. Indeed, since $y_{t}^{n} \rightarrow y_{t}$ strongly in $L^{2}\left(\Sigma_{0}\right)$ (see (3.15)), $y_{t}^{n}(x, t) \rightarrow y_{t}(x, t)$ a.e. in $\Sigma_{0}$. Let $\eta>0$. Using the theorem of Lusin and Egoroff, we can choose a subset $\omega \subset \Sigma_{0}$ such that $|\omega|<\eta, y_{t} \in L^{2}\left(\Sigma_{0} \backslash \omega\right)$ and $y_{t}^{n} \rightarrow y_{t}$ uniformly on $\Sigma_{0} \backslash \omega$. Thus, for each $\epsilon>0$, there is an $M>\frac{2}{\epsilon}$ such that

$$
\left|y_{t}^{n}(x, t)-y_{t}(x, t)\right|<\frac{\epsilon}{2} \text { for } n>M \text { and }(x, t) \in \Sigma_{0} \backslash \omega .
$$

Then, if $\left|y_{t}^{n}(x, t)-s\right|<\frac{1}{n}$, we have $\left|y_{t}(x, t)-s\right|<\epsilon$ for all $n>M$ and $(x, t) \in \Sigma_{0} \backslash \omega$. Therefore we have

$$
\underline{b}_{\epsilon}\left(y_{t}(x, t)\right) \leq b^{n}\left(y_{t}^{n}(x, t)\right) \leq \bar{b}_{\epsilon}\left(y_{t}(x, t)\right), \quad \forall n>M,(x, t) \in \Sigma_{0} \backslash \omega .
$$

Let $\phi \in L^{2}\left(0, T ; L^{2}\left(\Gamma_{0}\right)\right), \phi \geq 0$. Then

$$
\begin{aligned}
\int_{\Sigma_{0} \backslash \omega} \underline{b}_{\epsilon}\left(y_{t}(x, t)\right) \phi(x, t) d x d t & \leq \int_{\Sigma_{0} \backslash \omega} b^{n}\left(y_{t}^{n}(x, t)\right) \phi(x, t) d x d t \\
& \leq \int_{\Sigma_{0} \backslash \omega} \bar{b}_{\epsilon}\left(y_{t}(x, t)\right) \phi(x, t) d x d t
\end{aligned}
$$

Letting $n \rightarrow \infty$ in this inequality and using the last convergence result in (3.13), we obtain

$$
\begin{aligned}
\int_{\Sigma_{0} \backslash \omega} \underline{b}_{\epsilon}\left(y_{t}(x, t)\right) \phi(x, t) d x d t & \leq \int_{\Sigma_{0} \backslash \omega} \Xi(x, t) \phi(x, t) d x d t \\
& \leq \int_{\Sigma_{0} \backslash \omega} \bar{b}_{\epsilon}\left(y_{t}(x, t)\right) \phi(x, t) d x d t .
\end{aligned}
$$

Letting $\epsilon \rightarrow 0^{+}$in this inequality, we deduce that

$$
\Xi(x, t) \in \varphi\left(y_{t}(x, t)\right) \text { a.e. in } \Sigma_{0} \backslash \omega,
$$

and letting $\eta \rightarrow 0^{+}$we get

$$
\Xi(x, t) \in \varphi\left(y_{t}(x, t)\right) \text { a.e. in } \Sigma_{0} .
$$


It remains to show that $\zeta=A y$. From the approximated problem and the convergence results $(3.13)-(3.16)$, we see that

$$
\begin{aligned}
& \limsup _{n \rightarrow \infty} \int_{0}^{T}\left\langle A y^{n}(t), y^{n}(t)\right\rangle d t \\
\leq & \left(y_{1}, y_{0}\right)-\left(y_{t}(T), y(T)\right)+\int_{0}^{T}\left(y_{t}(t), y_{t}(t)\right) d t-\frac{1}{2}\|\nabla y(T)\|^{2} \\
& +\frac{1}{2}\left\|\nabla y_{0}\right\|^{2}-\int_{0}^{T}(\Xi(t), y(t))_{\Gamma_{0}} d t+\int_{0}^{T}\left(|y(t)|^{m-2} y(t), y(t)\right)_{\Gamma_{0}} d t .
\end{aligned}
$$

On the other hand, it follows from (3.17) that

$$
\begin{aligned}
\int_{0}^{T}(\zeta(t), y(t)) d t= & \left(y_{1}, y_{0}\right)-\left(y_{t}(T), y(T)\right)+\int_{0}^{T}\left(y_{t}(t), y_{t}(t)\right) d t \\
& -\frac{1}{2}\|\nabla y(T)\|^{2}+\frac{1}{2}\left\|\nabla y_{0}\right\|^{2}-\int_{0}^{T}(\Xi(t), y(t))_{\Gamma_{0}} d t \\
& +\int_{0}^{T}\left(|y(t)|^{m-2} y(t), y(t)\right)_{\Gamma_{0}} d t
\end{aligned}
$$

Combining (3.20) and (3.21), we get

$$
\limsup _{n \rightarrow \infty} \int_{0}^{T}\left\langle A y^{n}(t), y^{n}(t)\right\rangle d t \leq \int_{0}^{T}\langle\zeta(t), y(t)\rangle d t .
$$

Since $A$ is a monotone operator, we have

$$
\begin{aligned}
0 & \leq \limsup _{n \rightarrow \infty} \int_{0}^{T}\left\langle A y^{n}(t)-A z(t), y^{n}(t)-z(t)\right\rangle d t \\
& \leq \int_{0}^{T}\langle\zeta(t)-A z(t), y(t)-z(t)\rangle d t, \quad \forall z \in L^{2}(0, T ; V) .
\end{aligned}
$$

By Mintiy's monotonicity argument (see e.g. [17]),

$$
\zeta=A y \text { in } L^{2}\left(0, T ; V^{*}\right) .
$$

Therefore the proof of Theorem 2.1 is completed.

\section{Asymptotic behavior of solutions}

In this section we shall prove the decay rates (2.5) and (2.6) in Theorem 2.2 by applying Lemma 2.1. To prove the decay property, we first obtain uniform estimates for the approximated energy

$$
E^{n}(t)=\frac{1}{2}\left\|y_{t}^{n}(t)\right\|^{2}+\frac{1}{p}\left\|\nabla y^{n}(t)\right\|_{p}^{p}-\frac{1}{m}\left\|y^{n}(t)\right\|_{m, \Gamma_{0}}^{m}
$$

and then pass to the limit. Note that $E^{n}(t)$ is non-negative and uniformly bounded. Let us fix an arbitrary $t>0$. From the approximated problem (3.2) 
and $w_{j}=y_{t}^{n}(t)$, we get

$$
\frac{d}{d t} E^{n}(t)+\left\|\nabla y_{t}^{n}(t)\right\|^{2}=-\left(b^{n}\left(y_{t}^{n}(t)\right), y_{t}^{n}(t)\right)_{\Gamma_{0}} \leq-\mu_{1}\left\|y_{t}^{n}(t)\right\|_{\Gamma_{0}}^{2} .
$$

This implies that $E^{n}(t)$ is a nonincreasing function. Setting $F_{n}^{2}(t)=E^{n}(t)-$ $E^{n}(t+1)$ and integrating (4.1) over $(t, t+1)$ we have

$$
F_{n}^{2}(t) \geq \int_{t}^{t+1}\left(\left\|\nabla y_{t}^{n}(s)\right\|^{2}+\mu_{1}\left\|y_{t}^{n}(s)\right\|_{\Gamma_{0}}^{2}\right) d s
$$

By applying the Mean value theorem, there exist $t_{1} \in\left[t, t+\frac{1}{4}\right]$ and $t_{2} \in$ $\left[t+\frac{3}{4}, t+1\right]$ such that

$$
\left\|y_{t}^{n}\left(t_{i}\right)\right\|_{\Gamma_{0}} \leq \frac{2}{\sqrt{\lambda_{1}+\mu_{1}}} F_{n}(t), \quad i=1,2
$$

where $\lambda_{1}$ is a constant satisfying $\|z\|_{\Gamma_{0}}^{2} \leq \lambda_{1}\|\nabla z\|^{2}$ for $z \in V$.

Now, replacing $w_{j}$ by $y^{n}(t)$ in the approximated problem we have

$$
\begin{aligned}
& \left\langle A y^{n}(t), y^{n}(t)\right\rangle-\left(\left|y^{n}(t)\right|^{m-2} y^{n}(t), y^{n}(t)\right)_{\Gamma_{0}} \\
= & -\left(y_{t t}^{n}(t), y^{n}(t)\right)-\left(\nabla y_{t}^{n}(t), \nabla y^{n}(t)\right)-\left(b^{n}\left(y_{t}^{n}(t)\right), y^{n}(t)\right)_{\Gamma_{0}} .
\end{aligned}
$$

Integrating this over $\left(t_{1}, t_{2}\right)$ and using (4.1) and $\left(H_{1}\right)$, we get

$$
\begin{aligned}
& \int_{t_{1}}^{t_{2}} \frac{1}{p}\left\|\nabla y^{n}(s)\right\|_{p}^{p} d s-\int_{t_{1}}^{t_{2}}\left\|y^{n}(s)\right\|_{m, \Gamma_{0}}^{m} d s \\
\leq & \int_{t_{1}}^{t_{2}}\left\|\nabla y^{n}(s)\right\|_{p}^{p} d s-\int_{t_{1}}^{t_{2}}\left\|y^{n}(s)\right\|_{m, \Gamma_{0}}^{m} d s \\
= & -\left(y_{t}^{n}\left(t_{2}\right), y^{n}\left(t_{2}\right)\right)+\left(y_{t}^{n}\left(t_{1}\right), y^{n}\left(t_{1}\right)\right)+\int_{t_{1}}^{t_{2}}\left\|y_{t}^{n}(s)\right\|^{2} d s \\
& -\int_{t_{1}}^{t_{2}}\left(\nabla y_{t}^{n}(s), \nabla y^{n}(s)\right) d s-\int_{t_{1}}^{t_{2}}\left(b^{n}\left(y_{t}^{n}(s)\right), y^{n}(s)\right)_{\Gamma_{0}} d s \\
\leq & \left\|y_{t}^{n}\left(t_{2}\right)\right\|\left\|y^{n}\left(t_{2}\right)\right\|+\left\|y_{t}^{n}\left(t_{1}\right)\right\|\left\|y^{n}\left(t_{1}\right)\right\|+\int_{t_{1}}^{t_{2}}\left\|y_{t}^{n}(s)\right\|^{2} d s \\
& +c \int_{t_{1}}^{t_{2}}\left\|\nabla y_{t}^{n}(s)\right\|\left(\sup _{t \leq s \leq t+1}\left\|\nabla y^{n}(s)\right\|_{p}\right) d s+\mu_{2} \int_{t_{1}}^{t_{2}}\left\|y_{t}^{n}(s)\right\|_{\Gamma_{0}}\left\|y^{n}(s)\right\|_{\Gamma_{0}} d s .
\end{aligned}
$$

Using Holder's inequality, Poincare inequality and Eqs. (4.2)-(4.4), we get

$$
\begin{aligned}
& \int_{t_{1}}^{t_{2}} E^{n}(s) d s \\
= & \frac{1}{2} \int_{t_{1}}^{t_{2}}\left\|y_{t}^{n}(s)\right\|^{2} d s+\frac{1}{p} \int_{t_{1}}^{t_{2}}\left\|\nabla y^{n}(s)\right\|_{p}^{p} d s-\frac{1}{m} \int_{t_{1}}^{t_{2}}\left\|y^{n}(s)\right\|_{m, \Gamma_{0}}^{m} d s \\
\leq & c F_{n}^{2}(t)+c F_{n}(t)\left\{\left\|\nabla y^{n}\left(t_{2}\right)\right\|_{p}+\left\|\nabla y^{n}\left(t_{1}\right)\right\|_{p}+\sup _{t \leq s \leq t+1}\left\|\nabla y^{n}(s)\right\|_{p}\right\}
\end{aligned}
$$




$$
+\left(1-\frac{1}{m}\right) \int_{t_{1}}^{t_{2}}\left\|y^{n}(s)\right\|_{m, \Gamma_{0}}^{m} d s
$$

From (2.2) and the facts that $\left\|\nabla y^{n}(t)\right\|_{p}^{p} \leq \frac{m p}{m-p} E^{n}(t)$ and $E^{n}(t)$ is a nonincreasing function, it follows that

$$
\begin{aligned}
\left(1-\frac{1}{m}\right) \int_{t_{1}}^{t_{2}}\left\|y^{n}(s)\right\|_{m, \Gamma_{0}}^{m} d s & \leq\left(1-\frac{1}{m}\right) c_{*}^{m} \int_{t}^{t+1}\left\|\nabla y^{n}(s)\right\|_{p}^{m-p}\left\|\nabla y^{n}(s)\right\|_{p}^{p} d s \\
& \leq\left(1-\frac{1}{m}\right) c_{*}^{m}\left(\frac{2 m p}{m-p} E(0)\right)^{(m-p) / p} \frac{m p}{m-p} E^{n}(t)
\end{aligned}
$$

and hence we derive that

$$
\int_{t_{1}}^{t_{2}} E^{n}(s) d s \leq c F_{n}^{2}(t)+c F_{n}(t) E^{n}(t)^{1 / p}+C_{1} E^{n}(t)
$$

where $C_{1}=\left(1-\frac{1}{m}\right) c_{*}^{m}\left(\frac{2 m p}{m-p} E(0)\right)^{(m-p) / p} \frac{m p}{m-p}$. Young's inequality implies that

$$
\int_{t_{1}}^{t_{2}} E^{n}(s) d s \leq c F_{n}^{2}(t)+C_{\eta} F_{n}(t)^{p /(p-1)}+\frac{1}{\eta} E^{n}(t)+C_{1} E^{n}(t) .
$$

Noting that $E^{n}(t+1) \leq 2 \int_{t_{1}}^{t_{2}} E^{n}(s) d s$ and $E^{n}(t+1)=E^{n}(t)-F_{n}^{2}(t)$, we have from (4.6) that

$$
\left(\frac{1}{2}-C_{1}-\frac{1}{\eta}\right) E^{n}(t) \leq\left(c+\frac{1}{2}\right) F_{n}^{2}(t)+C_{\eta} F_{n}(t)^{p /(p-1)} .
$$

By the assumption (2.4), $\frac{1}{2}-C_{1}>0$ and hence taking $\eta>0$ is sufficiently small such that $\frac{1}{2}-C_{1}-\frac{1}{\eta}>0$, we obtain that

$$
E^{n}(t) \leq c F_{n}^{2}(t)+c F_{n}(t)^{p /(p-1)} .
$$

If $p=2$, then $E^{n}(t) \leq c F_{n}^{2}(t)$ and since $E^{n}(t)$ is decreasing from Lemma 2.1 there exist positive constants $C$ and $\gamma$ such that

$$
E^{n}(t) \leq C \exp (-\gamma t), \quad \forall t \geq 0 .
$$

If $p>2$, then Eq.(4.7) and the boundedness of $F_{n}(t)$ imply that

$$
E^{n}(t) \leq c F_{n}(t)^{p /(p-1)}
$$

and then

$$
E^{n}(t)^{2(p-1) / p} \leq c^{2(p-1) / p}\left(E^{n}(t)-E^{n}(t+1)\right) .
$$

Applying Lemma 2.1 to $\beta=\frac{p-2}{p}$, we obtain a constant $C>0$ such that

$$
E^{n}(t) \leq C(1+t)^{-p /(p-2)}, \forall t \geq 0 .
$$

Passing to the limit $n \rightarrow \infty$ in (4.8) and (4.9) we get Eqs. (2.5) and (2.6). This completes the proof of Theorem 2.2. 


\section{References}

[1] R. Adams, Sobolev Spaces, Academic Press, New York, 1975.

[2] S. Carl and S. Heikkila, Existence results for nonlocal and nonsmooth hemivariational inequalities, Journal of Inequalities and Applications 2006 (2006), Article ID 79532, 13 pages.

[3] J. L. Lions, Quelques méthodes de résolution des problèmes aux limites non linéaires, Dunod-Gauthier Villars, Paris, 1969.

[4] L. Liu and M. Wang, Global existence and blow-up of solutions for some hyperbolic systems with damping and source terms, Nonlinear Analysis 64 (2006), 69-91.

[5] S. A. Messaoudi, Global existence and nonexistence in a system of Petrovsky, J. Math. Anal. Appl. 265 (2002), 296-308.

[6] M. Miettinen, A parabolic hemivariational inequality, Nonlinear Anal. 26 (1996), 725734.

[7] M. Miettinen and P. D. Panagiotopoulos, On parabolic hemivariational inequalities and applications, Nonlinear Anal. 35 (1999), 885-915.

[8] M. Nakao, $A$ difference inequality and its application to nonlinear evolution equations, J. Math. Soc. Japan 30 (1978), 747-762.

[9] _ Energy decay for the quasilinear wave equation with viscosity, Math. Z. 219 (1995), 289-299.

[10] K. Ono, On global solutions and blow-up solutions of nonlinear Kirchhoff strings with nonlinear dissipation, J. Math. Anal. Appl. 216 (1997), 321-342.

[11] P. D. Panagiotopoulos, Inequality Problems in Mechanics and Applicatons. Convex and Nonconvex Energy Functions, Birkhäuser, Basel, Boston, 1985.

[12] _ Modelling of nonconvex nonsmooth energy problems. Dynamic hemivariational inequalities with impact effects, J. Comput. Appl. Math. 63 (1995), 123-138.

[13] J. Y. Park and J. J. Bae, On the existence of solutions of the degenerate wave equations with nonlinear damping terms, J. Korean Math. Soc. 35 (1998), 465-489.

[14] J. Y. Park, H. M. Kim, and S. H. Park, On weak solutions for hyperbolic differential inclusion with discontinuous nonlinearities, Nonlinear Anal. 55 (2003), 103-113.

[15] J. Y. Park and S. H. Park, On solutions for a hyperbolic system with differential inclusion and memory source term on the boundary, Nonlinear Anal. 57 (2004), 459-472.

[16] J. Rauch, Discontinuous semilinear differential equations and multiple valued maps, Proc. Amer. Math. Soc. 64 (1977), 277-282.

[17] R. E. Showalter, Monotone Operators in Banach Space and Nonlinear Partial Differential Equations, Mathematical Surveys and Monographs, 49, American Mathematical Society, Providence, RI, 1996.

[18] G. Todorova, Stable and unstable sets for the Cauchy problem for a nonlinear wave equation with nonlinear damping and source terms, J. Math. Anal. Appl. 239 (1999), $213-226$.

[19] C. Varga, Existence and infinitely many solutions for an abstract class of hemivariational inequlaities, Journal of Inequalities and Applications 2005 (2005), 89-105.

JiN MUN JEONG

Division of Mathematical Sciences

Pukyong National University

BUSAN 608-737, KOREA

$E$-mail address: jmjeong@pknu.ac.kr

JoNg YeOUl PARK

DePaRtMent of Mathematics

Pusan National University

BUSAN 609-735, Korea 
Sun Hye Park

Basic Sciences Research Institute

Pukyong National University

BUSAN 608-737, KOREA

E-mail address: sh-park@pusan.ac.kr 